\title{
New electrical parameters extraction method based on simplified 3D model using finite element analysis
}

\author{
Jorge Rafael González-Teodoro and \\ Enrique González Romero-Cadaval \\ Department of Electrical, Electronic and Control Engineering, \\ School of Industrial Engineering, University of Extremadura, Badajoz, Spain \\ Rafael Asensi and Roberto Prieto \\ Polytechnic University of Madrid, Madrid, Spain, and \\ Vladimir Kindl \\ Faculty of Electrical Engineering, University of West Bohemia, \\ Pilsen, Czech Republic
}

\begin{abstract}
Purpose The purpose of this paper is the presentation of an electrical equivalent circuit for inductive components as well as the methodology for electrical parameter extraction by using a 3D finite element analysis (FEA) tool.

Design/methodology/approach A parameter extraction based on energies has been modified for three dimensions. Some simplifications are needed in a real model to make the $3 \mathrm{D}$ finite element method (FEM) analysis operative for design engineers. Material properties for the components are modified at the pre modeling step and a corrector factor is used at the post modeling step to achieve the desired accuracy.

Findings The current hardware computational limitations do not allow the 3D FEA for every magnetic component, and due to the component asymmetries, the $2 \mathrm{D}$ analysis are not precise enough. The application of the new methodology for three dimensions to several actual components has shown its usefulness and accuracy. Details concerning model parameters extration are presented with simulation and measurement results at different operation frequencies from $1 \mathrm{kHz}$ to $1 \mathrm{GHz}$ being the range of switching frequencies used by power electronic converters based on $\mathrm{Si}, \mathrm{SiC}$ or $\mathrm{GaN}$ semiconductors.

Practical implications This new model includes the high frequency effects (skin effect, proximity effect, interleaving and core gap) and other effects can be only analyzed in 3D analysis for non symmetric components. The electrical parameters like resistance and inductance (self and mutual ones) are frequency dependent; thus, the model represents the frequency behavior of windings in detail. These parameters determine the efficiency for the inductive component and operation capabilities for the power converters (as in the voltage boost factor), which define their success on the market.

Originality/value The user can develop 3D finite element method (FEM) based analyses with geometrical simplifications, reducing the CPU time and extracting electrical parameters. The corrector factor presented in this paper allows obtaining the electrical parameters when 3D FE simulation would have

This research has been supported by the Junta de Extremadura, under project IB18067 and by the Ministry of Education, Youth and Sports of the Czech Republic under the project OP VVV Electrical Engineering Technologies with High Level of Embedded Intelligence CZ.02.1.01/0.0/0. 0/18 069/ 0009855 and by funding program of the University of West Bohemia number SGS2018 009.
\end{abstract}


developed without any geometry simplications. The contribution permits that the simulations do not need a high computational resource, and the simulation times are reduced drastically. Also, the reduced CPU time needed per simulation gives a potential tool to optimize the non symmetric components with $3 \mathrm{D}$ FEM analysis.

Keywords Electromagnetism, Finite element analysis, Power electronic simulation, Computational electromagnetics, Magnetic equivalent circuit, Power electronic devices modeling, Magnetics, 3D modeling, Transformers, Resistance, Inductance

Paper type Research paper

\section{Introduction}

Power electronics engineers need to use inductive components as an important part of their converters. The electrical parameters for the inductor have a critical influence in the overall efficiency and operation capabilities for the power converter.

The calculation of the winding resistance and inductance could be conducted by application the Maxwell"s equations. Nevertheless, the complete analytical solution for these equations has only been available for components with $1 \mathrm{D}$ magnetic field distribution.

Another alternative is to obtain the winding parameters by laboratory testing, but the component design or optimization could be expensive and time consuming.

A finite element analysis (FEA) is an adequate tool to calculate the winding parameters of any asymmetrical magnetic components (Iatcheva et al., 2018), but the current hardware computational limitations (the Random Access Memory is usually the limiting factor) do not natively allow to perform very complex $3 \mathrm{D}$ simulation considering high-frequency effects, interleaved winding or assembling air gap.

On the other hand, the calculation based on $1 \mathrm{D}$ or 2D FEA is not applicable due to the high degree of geometrical simplification. The only possible way for this type of calculation is to significantly reduce the number of mesh elements and perform complex $3 \mathrm{D}$ simulation.

Several various approaches for electrical parameters extraction have been developed based on FEA optimization (Okamoto et al., 2016; Kiselev et al., 2016), original methodologies (Ben Messaoud et al., 2016) or for particular components (Aljohani et al., 2016; Phukan et al., 2016; Niyomsatian et al., 2018; Lu and Ngo, 2017) without present an actual $3 \mathrm{D}$ model of the magnetic non-symmetrical component including all effects (skin effect, proximity effect, interleaving, core air gap).

In this work, the attention is paid mainly to the components with $\mathrm{EE}$ and toroidal magnetic cores (non-symmetrical coil configurations) because they are common for inductors, lack $3 \mathrm{D}$ symmetry and the winding parameters (resistance and inductance) cannot be calculated either using $1 \mathrm{D}$ or $2 \mathrm{D}$ FEA. The asymmetry of these inductive components lies mainly in the square shape of the windings.

As shown in (Gonzalez-Teodoro et al., 2015; Coulomb, 2014), successful convergence of $3 \mathrm{D}$ simulation requires the adoption of certain simplifications that more or less affect the results.

The parameter extraction presented in this paper is based on an electrical equivalent circuit discussed in Asensi et al. (2007) and modified for three dimensions (Figure 1.) This equivalent circuit stands on the superposition theorem which generally introduces some limitations.

It is only applied on the magnetic fields and the current densities (not on energies or losses) when analyzing a linear system. The FEM simulation therefore considers a constant permeability and coercivity set for the magnetic core material (Eddy current solver used). In other words, the model is not applicable to the systems with non-linear saturation $(\mathrm{BH}$ curve). 
In Section 2, the different analyses, the parameter extraction procedure and research developed to calculate the correction factor will be explained. Section 3 will introduce the original methodology to be used by the power engineers. Section 4 is dedicated to the experimental validation for eight different inductive components and showing the CPU reduction time. Conclusions and advantages for the proposed procedure are given in Section 5.

\section{Analysis for non-symmetrical inductive components}

The procedure of obtaining the correction factor is based on elementary geometry FEM analyses (referring to cases I, II, III, IV) and proper mathematical regressions. The cases have been modeled with the geometry assistant in the FEA (Figure 2). The modeling has been done following next process:

- Selected solver has been the Eddy Current Type with a frequency in the range from $1 \mathrm{~Hz}$ to $1.2 \mathrm{GHz}$.

- Material properties of the core and windings have been adjusted using the model descriptor function

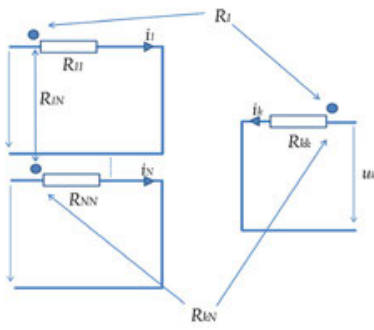

(a)

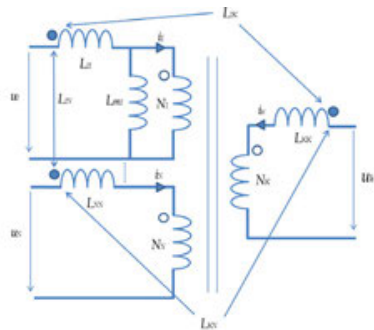

(b)

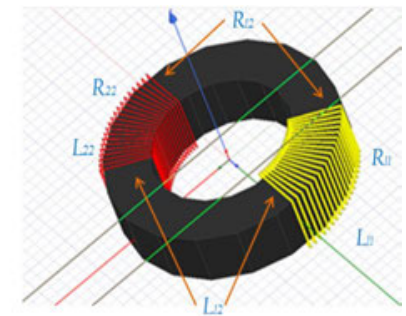

(c)
Figure 1.

Electrical equivalent circuit for non

symmetric components

Notes: (a) Generic Resistive Model; (b) generic magnetic model model; (c) example of 2 windings $3 \mathrm{D}$ component

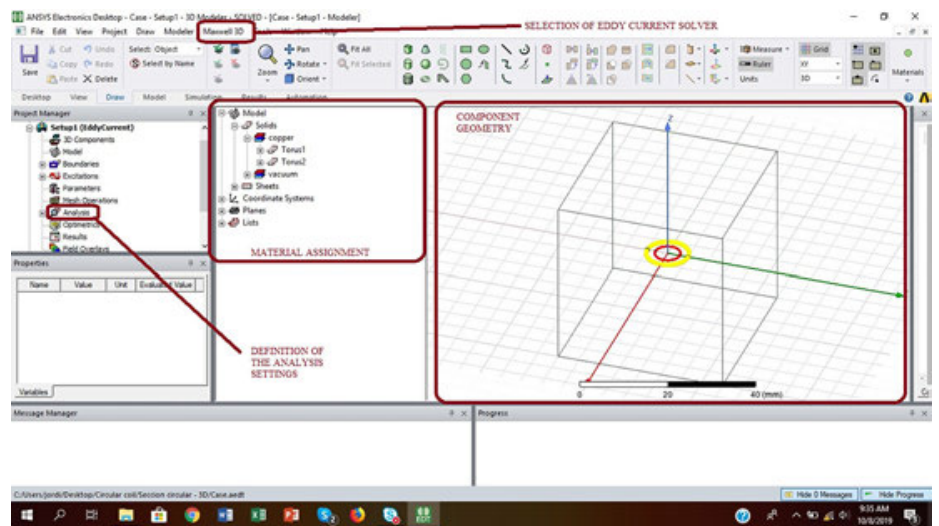

Figure 2. Ansys Maxwell environment for modeling and simulation of magnetic components 
- the mesh used for FEM analysis is automatic generated with these settings: 1 per cent error,1 25 maximum number of steps and step refinement of 20 per cent.

- Finally, the excitations (both, input and output signals) has been introduced using the sheets created into windings.

The material properties have been adjusted in the software database, the current density has been introduced according to the Transient solver and the mesh has been set up automatically. More detail about FEA methodology is given in Section 3.

\subsection{Polygonal model}

The conductor cross-sections for analyzed case studies are modified from a circular shape (original geometry) to a polygonal shape to reduce the FE number and achieve easier computational convergence. These modification change both the cross-section area and the distance between conductors which consequently affects the winding resistance and inductance. The material properties (1-2) must be accordingly tuned in the model to achieve the same resistance and inductance values that were measured for original geometry (Gonzalez-Teodoro et al., 2015):

$$
\begin{gathered}
\sigma_{\text {Pol }}=\frac{S_{\text {Circular }}}{S_{\text {Polygon }}} \cdot \sigma_{\text {Circular }} \\
\mu_{\text {Pol }}=\frac{S_{\text {Circular }}}{S_{\text {Polygon }}} \cdot \mu_{\text {Circular }}
\end{gathered}
$$

In (1) and (2), the parameters $\sigma_{P o l}$ and $\mu_{P o l}$ represent the conductivity and the permeability for the polygonal conductor, $\sigma_{\text {Circular }}$ and $\mu_{\text {Circular }}$ represent the same but for the circular conductor and $\mathrm{S}_{\text {Pircular }}$ and $\mathrm{S}_{\text {Polygonal }}$ stand for the cross-section areas of both geometrical variants respectively.

To minimize any distortion of the results, the zero vector potential boundary condition is set on the surface of enclose "free-space" region (having a size 5 times higher that of the component being analyzed) surrounding the conductor.

The analyzed inductive component is named "Polygonal Model" in the paper. The simulation is performed using Eddy Current Solver (Ansys Maxwell) with an energy error of 2 per cent and using standard defined mesh. The mesh is imported from a transformer (multiwinding component) to compare the results from different inductive components (single winding) having the same mesh.

At the post-processing stage, the parameter extraction is accomplished according to the magnetic-electrical equivalent circuit shown in Figure 1. The resistance and the inductance for the windings are calculated using a JAVA script based on equations (3) and (4) introduced in Asensi et al. (2007) and modified for three dimensions:

$$
\begin{aligned}
& L_{i j}=\frac{1}{I_{0}^{2}} \oiiint_{\mathrm{V}} \operatorname{Re}\left(\overrightarrow{\boldsymbol{B}}_{i 0} \cdot{\overrightarrow{\boldsymbol{H}^{*}}}_{j 0}\right) d v \\
& R_{i j}=\frac{1}{I_{0}^{2}} \oiiint_{\mathrm{V}} \operatorname{Re}\left(\overrightarrow{\boldsymbol{J}}_{i 0} \cdot{\overrightarrow{\boldsymbol{J}^{*}}}_{j 0}\right) d v
\end{aligned}
$$


where $I_{o}$ is the winding current (rms value), $\vec{B}$ is the magnetic flux density, $\overrightarrow{H^{*}}$ is the complex conjugate value of the magnetic field strength, and $\vec{J}$ is the current density, $\overrightarrow{J^{*}}$ is the complex conjugate of the current density. The indexes correspond to the mutual coefficients for the electrical parameters when there are more than two windings in the magnetic component.

For a wide supply frequency range, the current density distribution within the wire is affected by alternating internal and external electromagnetic fields. These phenomena are usually presented as a skin and proximity effect and have different behavior for circular and polygonal cross-section. Therefore, a correction factor $k$ must be applied to the extracted resistance $\left(R_{i j}^{F E M}\right)$ to get a proper resistance value. In this case the inductance is practically not influenced by the polygonal model and therefore the correction factor is not necessary (Zhang et al., 2017; Dias et al., 2018; Igarashi, 2017; Christian Bednarz et al., 2018).

\subsection{Correction factor depending on the frequency}

The correction factor [equation (6)] depending on the frequency is applied to the extracted resistance [equation (4)] introduced for the Polygonal Model:

$$
R_{i j}^{r e a l}=k \cdot R_{i j}^{F E M}
$$

where $R^{r e a l}$ is the resistance for the original model (circular cross-section) and $R^{F E M}$ is the resistance obtained from the Polygonal Model using equation (4). The correction factor is divided in two coefficients corresponding to the skin and proximity effects that depend on the frequency. According to Ferreira (1994), the skin and the proximity effect can be calculated separately due to the orthogonal relation existing between them:

$$
k=k_{\text {skin }}+k_{\text {proximity }}
$$

A large number of analysis have been performed to calculate the correction factor. It is based on the ratio of the resistance calculated for the real component and the resistance calculated for the Polygonal Model (7):

$$
E Q_{R}=\frac{R_{\text {cirle }}}{R_{\text {polygon }}}
$$

The correction factor also depends on the polygonal cross-section used in the simulation. A new term is introduced in the study to involve the polygonal cross-section dependency labeled $A_{p o l}$ and given in equation (8) (Figure 3):

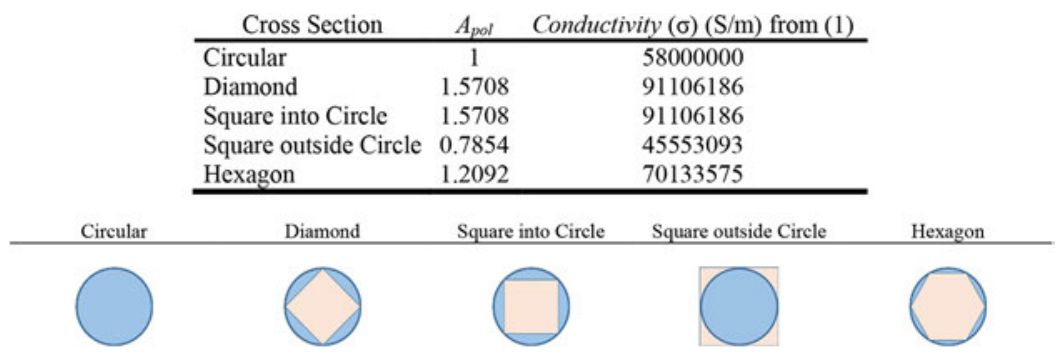

Figure 3. 


$$
A_{\text {pol }}=\frac{S_{\text {circle }}}{S_{\text {polygon }}}
$$

Equations (1) and (2) can be rewritten with the new term $A_{\text {pol }}$ as it is indicated in equations (9) and (10) (Figure 3):

$$
\begin{gathered}
\sigma_{\text {Pol }}=A_{p o l} \cdot \sigma_{\text {Circular }} \\
\mu_{\text {Pol }}=A_{p o l} \cdot \mu_{\text {Circular }}
\end{gathered}
$$

Figure 3 shows the conductivity settings for various shapes of conductor cross-sections areas. These material properties need to be used within the model solution.

\subsection{Analyses for the different cases}

2.3.1 Skin effect study (case I, II, III). Three simple cases have been studied to understand the skin in the polygonal cross-sections. The cases are a single wire alone (case I), a single circular coil (case II) and a single square coil (case III) which are shown in Figure 4 . The case studies have been developed for various conductor diameters $\left(\mathrm{R}_{1} \quad\right.$ AWG18, $R_{2} \quad$ AWG24, $\mathrm{R}_{3} \quad$ AWG14). The results of (7) calculated for single stand-alone wire (various radii) are presented in Figure 4. The solid lines show calculated values using FEA and the dashed lines represent their regression. As seen in Figure 5(a), a single stand-alone wire (case I) is affected only by the skin effect. The $E Q_{R}$ depends on the frequency and the conductor diameter. The other cases (II and III) have an influence from the current loop effect (interaction between the auto-proximity effect and the geometrical arrangement of the winding). As obvious from Figure 5(b), the circular coil introduces higher $E Q_{R}$ because of additional influence of the loop current effect. The $E Q_{R}$ for the circular coil introduces an additional parameter involved, the coil diameter. The results of (7) calculated for different cases of single square shaped coil including effects of ferromagnetic materials are presented in Figure 6. The solid lines show calculated values using FEA and the dashed lines represent their regression.

The analyzed geometries seen in Figure 6 (case III) exhibit deeper impact from the loop current effect. It is caused by the stronger external field that induces eddy currents into the wire either because of the smaller coil dimensions or because of the ferromagnetic material used. When the coil is wound on the ferromagnetic core, the current distribution inside the

Figure 4. Description of the three analyzed cases

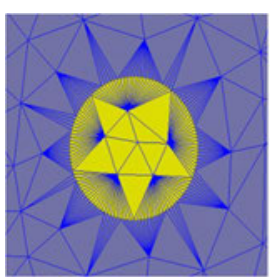

(a)

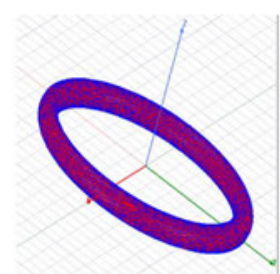

(b)

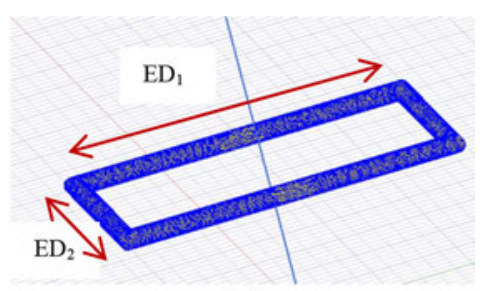

(c)
Notes: (a) A single wire alone (case I); (b) a single circular coil (case II); (c) a single square coil (case III) 


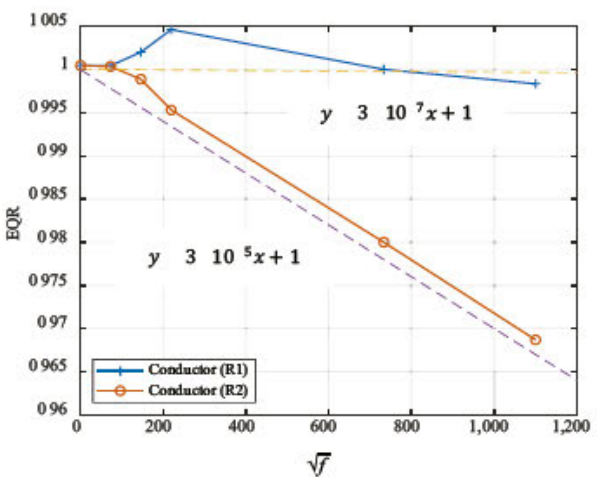

(a)

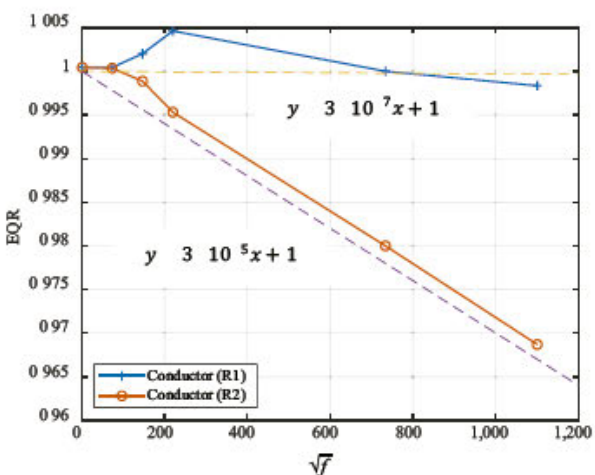

(b)

Notes: (a) $R_{I}$ (AWG18) and $R_{2}$ (AWG24). Single alone wire; (b) $R_{1}$ (AWG18) and $R_{2}$ (AWG24). Single circular coil

wire is quite different from the previous cases (principle of mirror images). To meet very high accuracy within this effect a number of permutations (see Table I - 305 cases analyzed) have been studied. The results for all $E Q_{R}$ are in agreement with the theoretical approach presented in Belevitch (1971).

From electromagnetic theory, the resistance for a circular conductor is defined in (11), and the skin factor for a circular section [equation (12)] is ruled by equation (13), (Zhang et al., 2017):

$$
\begin{gathered}
R=\frac{1}{\sigma} \frac{l}{S} \\
S_{\text {Circular }}=\pi \cdot r^{2} \\
\delta=\sqrt{\frac{2}{\omega \mu \sigma}}
\end{gathered}
$$

where $R$ is the resistance, $l$ is the average conductor length, $S$ is the conductor crosssection area, $r$ is the radius of the original wire (having circular cross-section), $\omega$ is the angular frequency and $\delta$ is the skin depth for circular sections. The cross-section area used for the resistance calculation is determined in equation (14) depending on the frequency driven by the skin effect. Thus, the resistance for the circular conductor is calculated by equation (15):

$$
S_{\text {real }}=\pi \cdot r_{1}^{2}-\pi \cdot\left(r_{1}-\delta\right)^{2}=2 \pi r \delta-\pi \delta^{2}
$$

Using (14) to calculate $E Q_{R}$ and introducing $\beta$ (skin depth for polygonal sections), equation (15) valid for $\delta \geq r$ is obtained: 


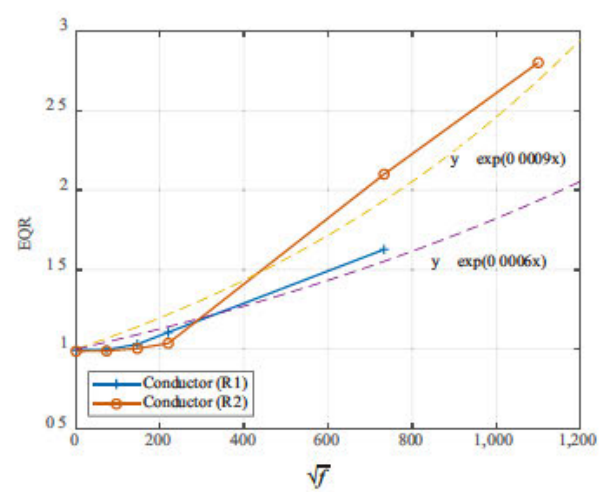

(a)

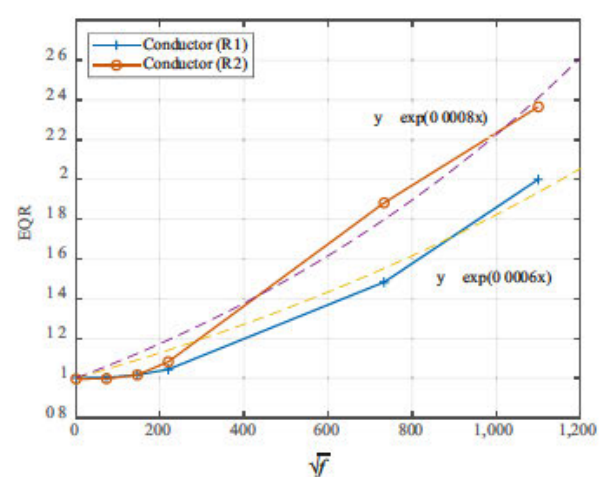

(c)

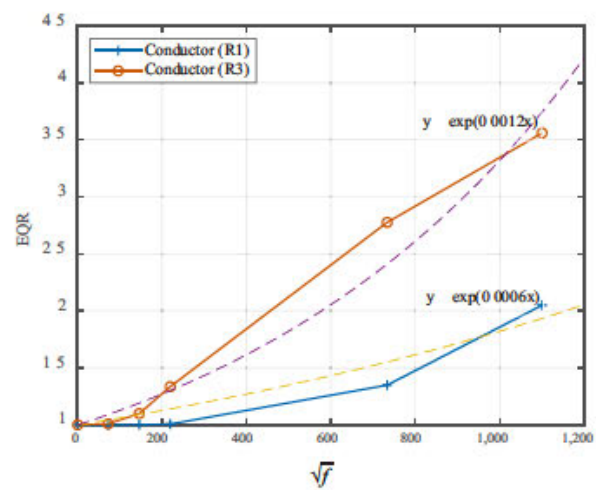

(e)

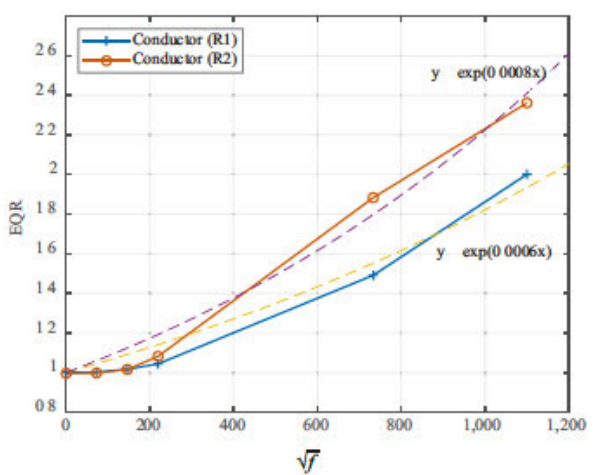

(b)

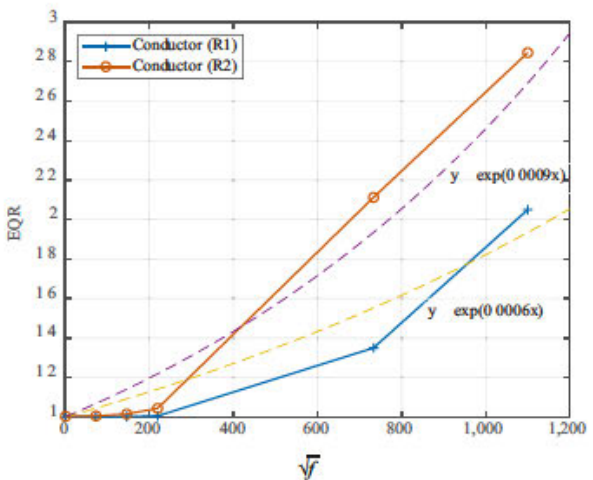

(d)

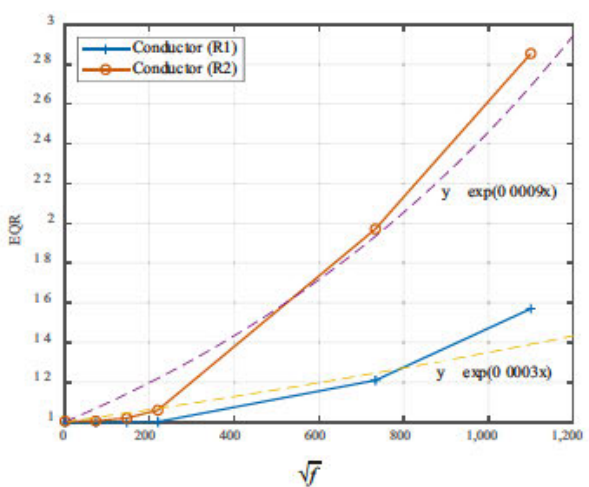

(f) single square coil (Polygonal Model with hexagonal section)

Notes: (a) Ferrite core (3C90); (b) Air core; (c) 4A driven in both conductors; (d) Square edge $E D_{1} \quad E D_{2} \quad 20 \mathrm{~mm}$; (e) $\mathrm{R}_{1} \quad A W G 18$ and $\mathrm{R}_{3} \quad$ AWG14; (f) Square edge $E D_{1} E D_{2} \quad 5 \mathrm{~mm}$ 


\begin{tabular}{|c|c|c|c|c|}
\hline $\begin{array}{l}\text { Parameter } 1 \\
\text { (Conductor cross section) }\end{array}$ & $\begin{array}{c}\text { Parameter } 2 \\
\text { (Conductor Diameter) }\end{array}$ & $\begin{array}{c}\text { Parameter 3a } \\
\left.\text { (Edge length } \mathrm{ED}_{1} *\right)\end{array}$ & $\begin{array}{c}\text { Parameter } 3 \mathrm{~b} \\
\left.\text { (Edge length } \mathrm{ED}_{2}{ }^{*}\right)\end{array}$ & $\begin{array}{c}\text { Parameter } 4 \\
\text { (Core Material) }\end{array}$ \\
\hline Circular & $\begin{array}{c}\text { AWG29 } \\
(d=0.286 \mathrm{~mm})\end{array}$ & Standard & Standard & Vacuum \\
\hline Diamond & $\begin{array}{c}\text { AWG24 } \\
(d=0.510 \mathrm{~mm})\end{array}$ & $+5 \mathrm{~mm}$ & $+5 \mathrm{~mm}$ & 3С90 \\
\hline Square into Circle & $\begin{array}{c}\text { AWG18 } \\
(d=1.02 \mathrm{~mm})\end{array}$ & $+10 \mathrm{~mm}$ & $+10 \mathrm{~mm}$ & 3С98 \\
\hline $\begin{array}{l}\text { Square outside Circle } \\
\text { Hexagon }\end{array}$ & $\operatorname{AWG14}(d=1.63 \mathrm{~mm})$ & $\begin{array}{l}+15 \mathrm{~mm} \\
+20 \mathrm{~mm}\end{array}$ & $\begin{array}{l}+15 \mathrm{~mm} \\
+20 \mathrm{~mm}\end{array}$ & $\begin{array}{c}3 \mathrm{~F} 4 \\
4 \mathrm{~F} 11\end{array}$ \\
\hline
\end{tabular}

Note: *See Figure 4

$$
E Q_{R}=\frac{R_{\text {Circular }}}{R_{\text {polygon }}}=\frac{\frac{l}{S_{\text {Circular }}}}{\frac{l}{S_{\text {polygonal }}}}=\frac{S_{\text {polygonal }}}{S_{\text {Circular }}}=\frac{2 \pi r \beta-\pi \beta^{2}}{2 \pi r \delta-\pi \delta^{2}}
$$

Therefore, according to results from all $E Q_{R}$ tendencies calculated for different permutations (case III), it could be assumed equation (16):

$$
\beta \leq \sqrt{\frac{2}{\omega \mu \sigma}}
$$

An arithmetical regression from all results (shown in Table I for case III) relating to single square coil, the $E Q_{R}$ corresponds to equation (17) and the according corrector factor for skin effect results equation (18):

$$
\begin{gathered}
E Q_{R}=A_{p o l} \cdot r^{1 / 2} \cdot f \\
k_{\text {skin }}=e^{\left(\frac{A_{p o l}}{1000} \cdot \frac{1}{2}\right) \cdot f}
\end{gathered}
$$

where $r$ is still the radius of the original coil wire having circular cross-section and $f$ is the frequency.

2.3.2 Proximity effect study (case IV). There are two well-known methods for calculation high-frequency effects in windings. The methods from Dowell (1966) and from Ferreira (1994) give significantly different results at high frequency. Both methods can have substantial errors, exceeding 60 per cent. The Ferreira method, which is based on the exact Bessel-function solution for the eddy current in an isolated conducting cylinder subjected to a time varying magnetic field, is found to be most accurate for loosely packed windings, whereas to Dowell method, which approximates winding layers comprising multiple turns of round wire with a rectangular conducting sheet, is most accurate for closely-packed windings.

The proximity effect will be analyzed in detail for the square coils (the windings used in the EE and Toroidal cores). Based on Dowell (1966) the skin effect and proximity effect can be calculated separately due to the orthogonality existing between them. A square shaped winding having different number of coil turns (case IV - 1 turn to 14 turns), have been 
studied (see Figure 7) while permuting all the geometrical parameters used in case III. By excluding the influence of the skin effect, calculated in the previous section (case III), one may write (19) for the proximity effect:

$$
k_{p r o x}=e^{\left(\frac{A_{p o l}}{1000} \cdot \frac{n}{k_{C}} \cdot r^{1 / 2}\right) \cdot f}
$$

For (19) the parameter $k_{C}$ can be find in Table II. It is a combined number without repetition $\left(\frac{m}{2}\right)$, where $m$ represents the number of edges of the polygonal cross-section and $n$ is the number of the turns in the winding. The factor $k_{C}$ takes into account various cross-section area chosen for studied conductors (important parameter for the proximity effect) in FEA using the real model or the Polygonal Model.

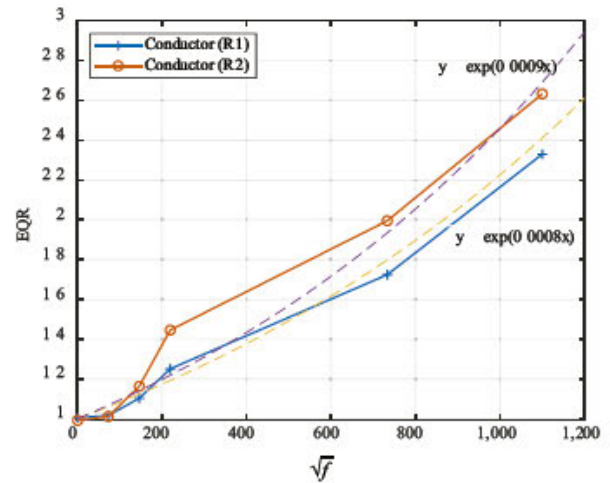

(a)

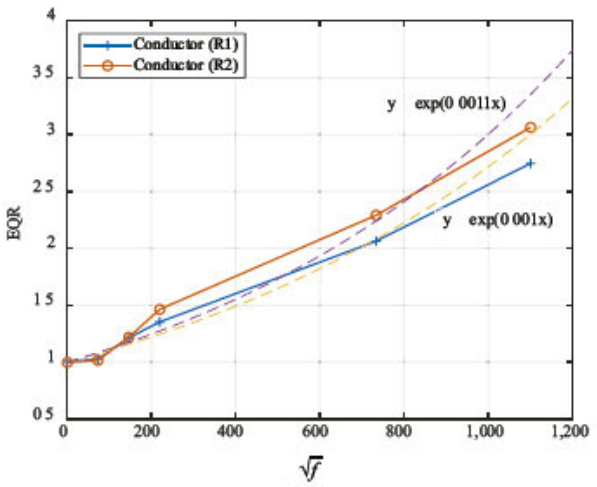

(c)

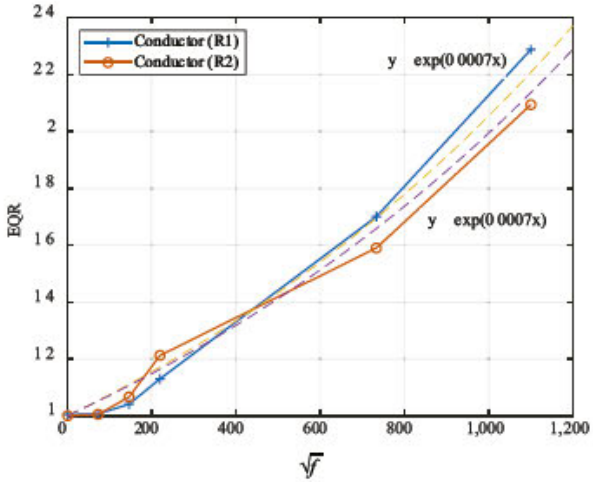

(b)

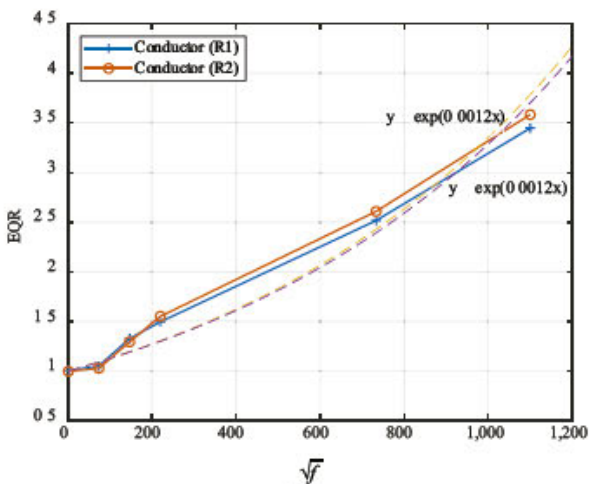

(d)

Notes: (a) 2 conductors in primary (R1) and 2 conductors in secondary ( $R 2$ ); (b) 4 conductors in primary $(R 1)$ and 4 conductors in secondary $(R 2)$; (c) 8 conductors in primary $(R 1)$ and 8 conductors in secondary $(R 2)$; (d) 14 conductors in primary $(R 1)$ and 14 conductors in secondary $(R 2)$ 
Table II shows the value of $k_{C}$ depending of the polygonal shape used for the winding crosssections in the Polygonal Model. The results of calculated for different cases are graphically presented in Figure 7. The solid lines show calculated values using FEA and the dashed lines represent their regression.

\section{Proposed methodology for power electronics designers}

This method is based on $3 \mathrm{D}$ FEA organized into three steps: pre-processing, simulation (processing) and post-processing.

(1) Pre-processing:

- The inductive component is modeled in 3D using the FEM software.

- The conductor cross-sections area is modified from circular to polygonal (hexagonal recommended) to reduce the $\mathrm{FE}$ number and to achieve better computational convergence.

- The zero vector potential boundary condition is set on the surface of enclose "free-space" region (having a size 5 times higher that of the component being analyzed) surrounding the conductor. The frequency operation range and the winding current are defined into the solution settings.

- The conductor material properties are modified according to (1-2).

(2) Simulation (processing):

- This procedure is completed due to the FE reduction using the Polygonal model. The Eddy Current Solver is used within the Ansys Maxwell tool using standard defined computational mesh.

(3) Post-processing:

- The electrical parameters of the equivalent circuit are extracted using (3-4) for the Polygonal Model. The inductance is accurate enough for low-high frequency range.

- The obtained resistance is only valid in very low frequency. At difference frequency range, the correction factor $(5)$ or $(18+19)$ must be applied to achieve accurate results.

\section{Validation}

The validation of proposed methodology has been accomplished with experimental measurements for 8 different non-symmetric inductive components. These inductive components have been connected to the impedance analyzer (4294 A) on the windings terminals in open circuit.

The analyzer is configured with the equivalent circuit shown in Figure 8 and is described by (20) and (21):

$$
Z_{c c 1}=R_{c c 1}+j X_{c c 1}
$$

\begin{tabular}{lccccccc}
\hline $\mathrm{m}$ & 4 & 6 & 8 & 10 & 12 & 18 & $\begin{array}{r}\text { Definition of } k_{C} \\
\text { depending on the } \\
\mathrm{k}_{\mathrm{C}}\end{array}$ \\
\hline
\end{tabular}




$$
Z_{01}=R_{01}+j X_{m 1}
$$

The equivalent circuit for the analyzer is different from the equivalent circuit used in the parameter extraction procedure using FEA based on energies. Therefore, the simplest way to validate the results is using equations (22) and (23) to transform the experimental measurements in comparable parameters with the parameters extracted from FEM based method:

$$
\text { Open Circuit Test } Z_{\mathrm{o} 1}+Z_{\mathrm{ccl}} \approx Z_{11}
$$

$$
\text { Short Circuit Test (example of } 2 \text { windings) } \quad Z_{\mathrm{cc} 1} \approx Z_{11}+a^{2} \cdot Z_{22}-2 a \cdot Z_{12}
$$

Here $Z_{01}$ and $Z_{c c 1}$ are the parameters from the traditional equivalent circuit and $a$ is the turn ratio. Thus, values from (23-24) are comparable to the values obtained from the proposed $\operatorname{method}\left(k \cdot R_{i j}^{F E M}\right)$.

\subsection{Component I}

Component I is a EE core (3C90 material) two-winding transformer. See Table III for the configuration and Figure 9 for the simulated and tested components. This component could not be simulated with Ansys Maxwell in a common PC with 4 GB of RAM due to its complex geometry, comprising a high number of circular conductors. The geometric model was simplified using a polygonal conductor (hexagonal conductors) shape as explained in Section 2 and this Polygonal Model needed a bit more than $3.12 \mathrm{~min}$ to converge. The methodology explained in Section 3 was used to calculate the winding resistances. Table IV shows the comparison between the measurement resistance and the estimated resistance for
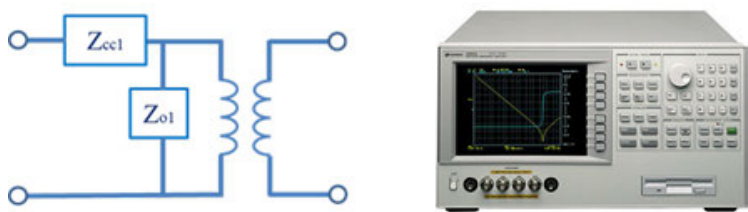

Figure 8.

Information about Impedance Analyzer
Notes: (a) Equivalent circuit from the analyzer

(b) Impedance analyzer (4294 A)

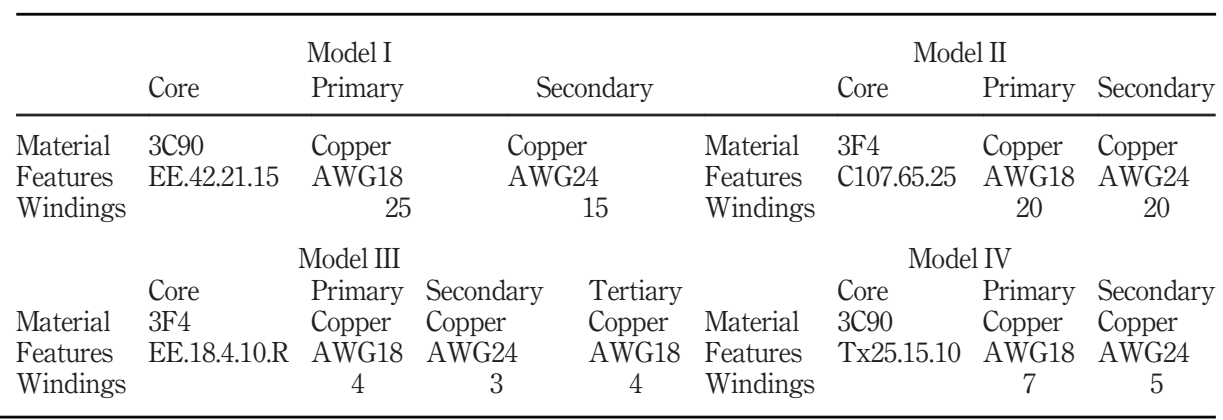

Table III.

Models information

Features
Windings 


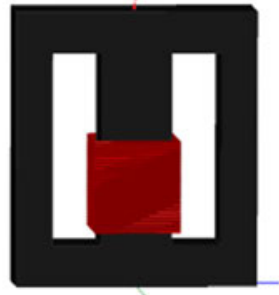

(a)

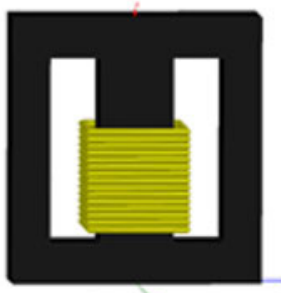

(b)

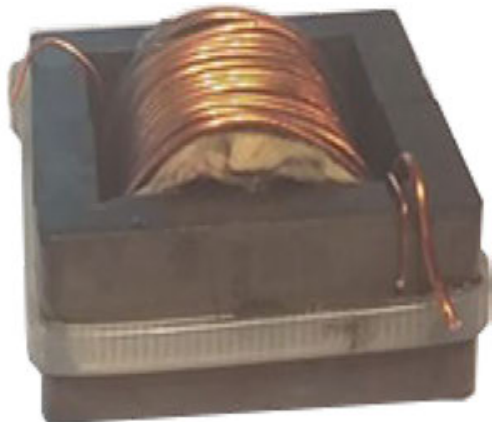

(c)

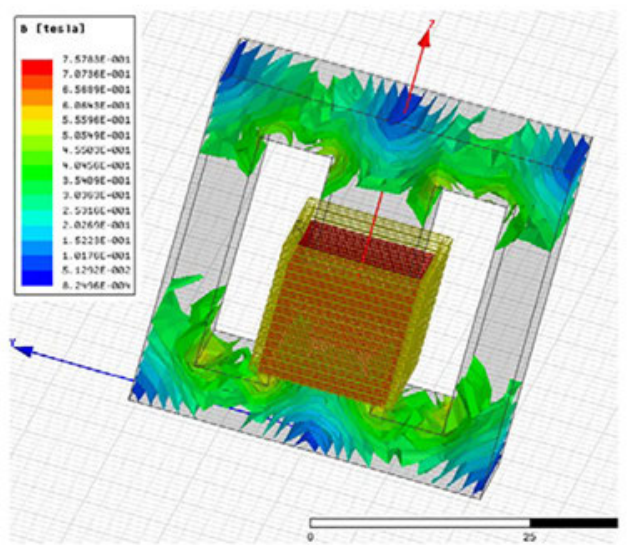

(d)

Figure 9.

FEM and tested model. component I

Notes: (a) Primary; (b) secondary; (c) tested component; (d) simulation results

the primary and secondary winding. (The results are expressed in percent with respect to the measured value.)

\subsection{Component II}

Component II is a Toroidal core ( $3 \mathrm{~F} 4$ material) two-winding transformer as shown in Figure 10. The details of the windings are indicated in Table III. Once again, the high number of turns did not allow the simulation with Ansys Maxwell in a 4 GB RAM either. The geometry was simplified using the Polygonal Model (hexagonal conductors), only 21,046 elements were needed, and allowed us to obtain the coefficients needed to estimate the power losses of the detailed component with the circular conductors. The experimental results and the estimated calculations of the winding resistances are shown in Table IV.

\subsection{Component III}

Component III is a three-winding transformer with an EE.18.4.10.R core (3F4 material). Table III and Figure 11 show the details of the simulated and tested models. This component has been chosen for two reasons: 


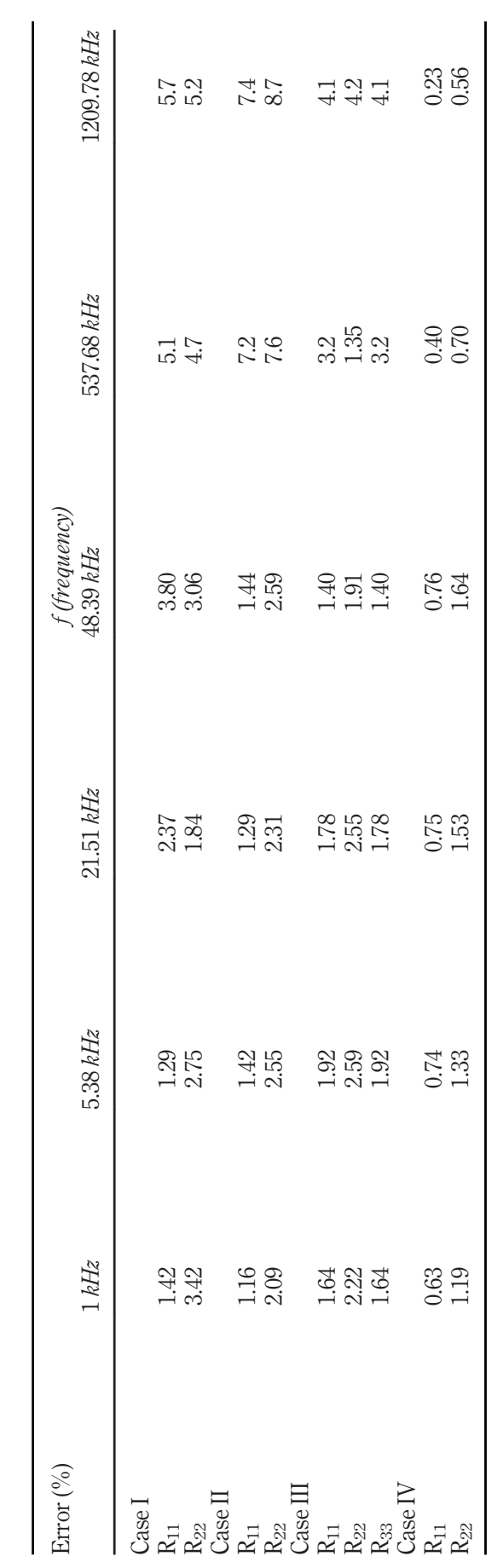

Resistances error $E$ (\%) $\quad(R)_{\text {real }} E(\%)$ $(R)_{\text {polygonal }} R_{\text {real }} \times$

100

Table IV. 


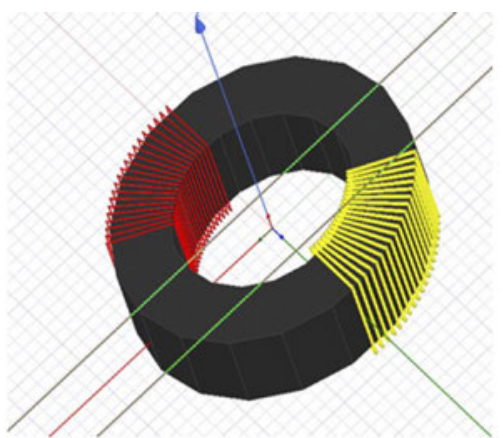

(a)

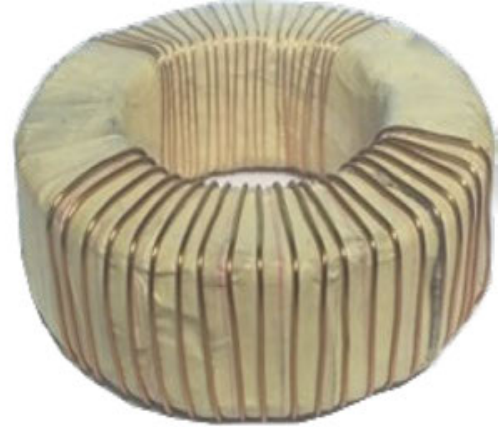

(b)

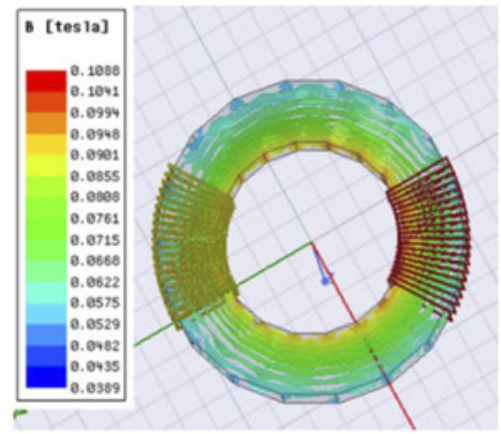

(c)

Figure 10.

FEM and tested model. component II

Notes: (a) FEM model; (b) tested component; (c) simulation results

(1) the component is a three winding model where the windings occupy all the core columns; and

(2) this model could be simulated with Ansys Maxwell with all its geometric complexity (no polygonal simplifications) which allows us to check the advantages of using this proposed methodology.

The simulation of the detailed model needed 662635 elements and converged in 59.89 minutes and the Polygonal Model (hexagonal conductors) needed just 34672 elements (a 5 per cent of the detailed simulation) and converged in 3.3 minutes of CPU time (a 6 per cent of the detailed simulation). Figure 13(a) shows the calculated results, detailed simulation, only the Polygonal Model simulation and resistance calculate with the Polygonal Model and correction factor. The experimental validation is indicated on Figure 13(c), where the sort circuit resistances are represented.

\subsection{Component IV}

Component IV is a TX25.15.10 (3C90 material). See Table III for the coil configuration. This component has been selected to check the benefit in CPU time. The model component is presented on Figure 12(a), the tested one is displayed on Figure 12(b) and the simulated 


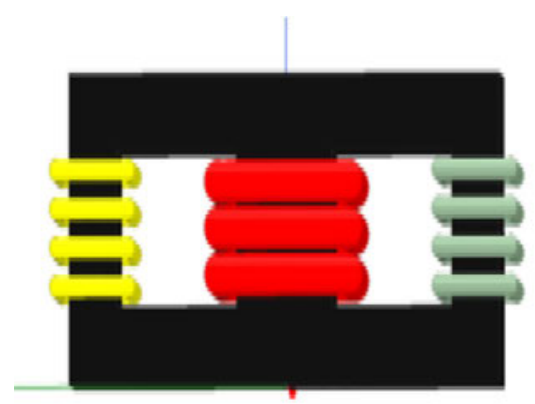

(a)

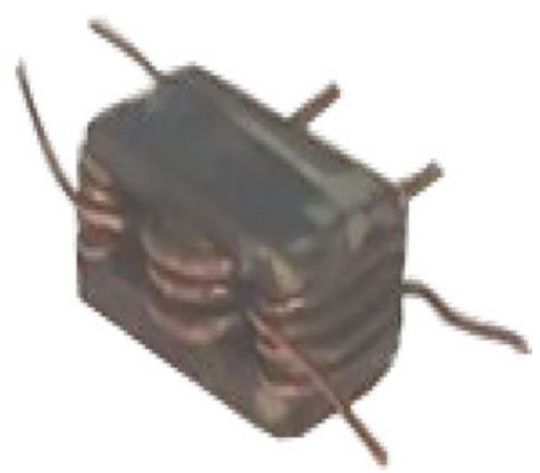

(b)
Figure 11.

Component III

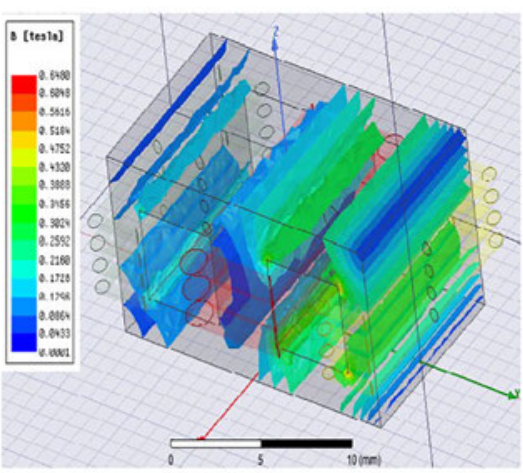

(c)

Notes: (a) FEM model; (b) tested component; (c) simulation results

results on Figure 12(c). The contrasting among the simulated values from the real component, the Polygonal one and the results using the corrector factor from the values of the Polygonal Model are shown on Figure 13(b) and the validations is indicated on Figure 13(d). The simulation of the original model has 445331 elements and was performed in $111.23 \mathrm{~min}$ and the Polygonal Model has had 5595 ( $<2$ per cent) FE and has been developed in $1.96(<2$ per cent) $\min$ (CPU time).

\subsection{Discussion}

Table IV summarizes the error for each component depending on the working frequency. The effect of the winding edge and the terminal connection has been removed to determinate the error between the proposed method and the performed experimental values. The analyzer measurements are similar to the simulation results obtained with the proposed method for all tested components up to low-medium frequencies $(\sim 500 \mathrm{kHz})$. The biggest error 8.7 per cent at $1.2 \mathrm{GHz}$ for case II, even in this case the error is much lower than the obtained with other known methods (Kiselev et al., 2016) applied to multi-winding components, that is close to 25 per cent. 


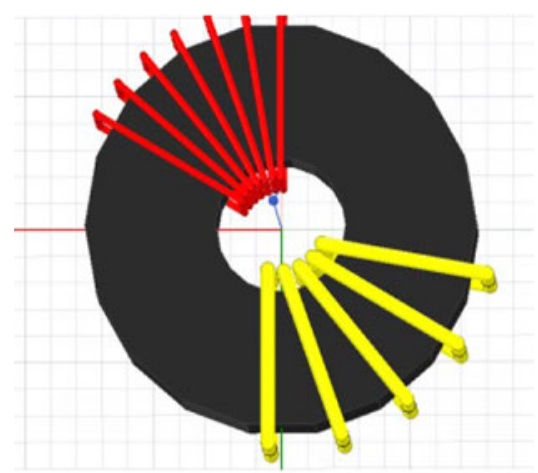

(a)

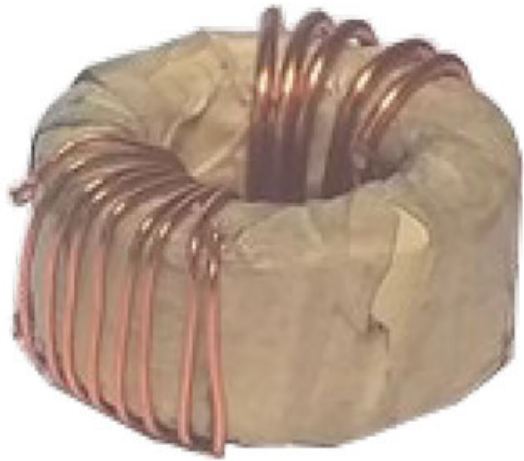

(b)

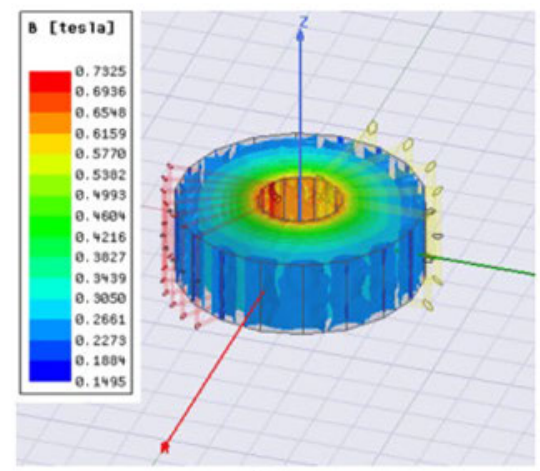

(c)

Figure 12. Component IV

Notes: (a) FEM model; (b) tested component; (c) simulation results

For the eight components, the FE number is reduced about 95 per cent from the real to the polygonal model, requiring a negligible computational time as compared to initial simulation using the real model without simplifications.

The values corresponding to the core magnetic field density (Figure 9(d), Figure 10(c), Figure 11(c) and Figure 12(c) for components I, II, III and IV respectively) obtained with the proposed method are very similar to the ones obtained with the simulation run without any simplification, but requiring much less CPU simulation time. The extrapolated thermal analysis will be are also valid using the simplified proposed method.

Figure 13 allows to conclude that a correction factor is required for the resistive parameter but no for the inductive parameter.

\section{Conclusions}

The determination of the resistance of an inductive component at power converters is one of the most critical steps for the electronics design because it is a limiter in the operational range for a power converter. 


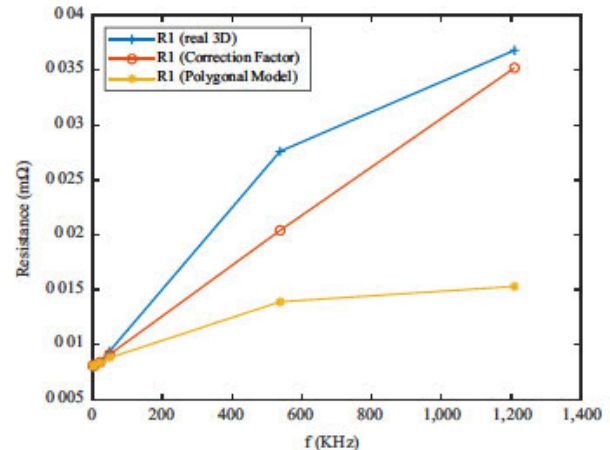

(a)

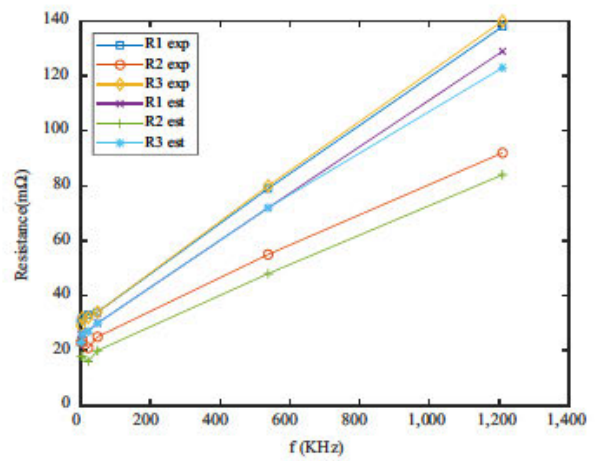

(c)

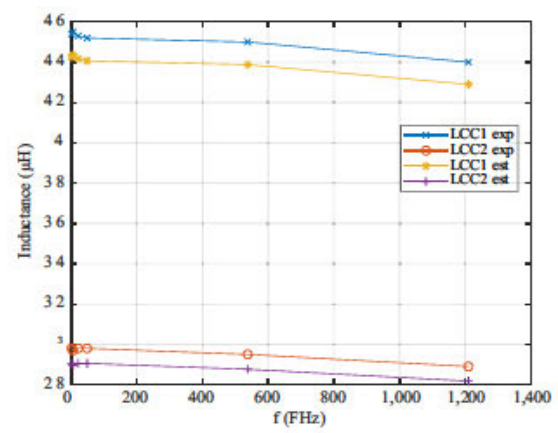

(e)

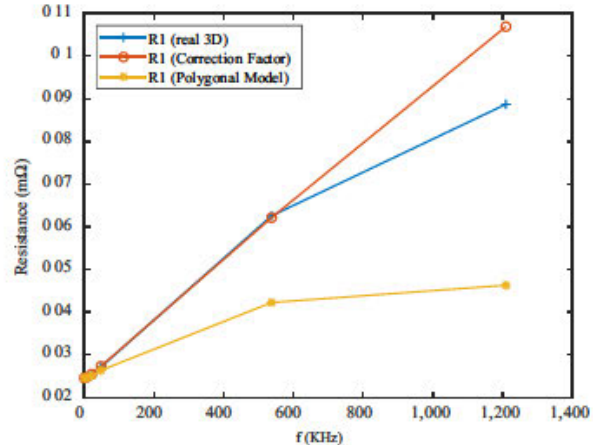

(b)

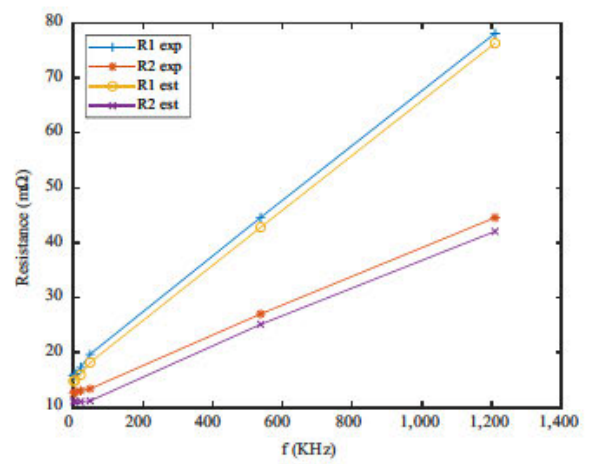

(d)

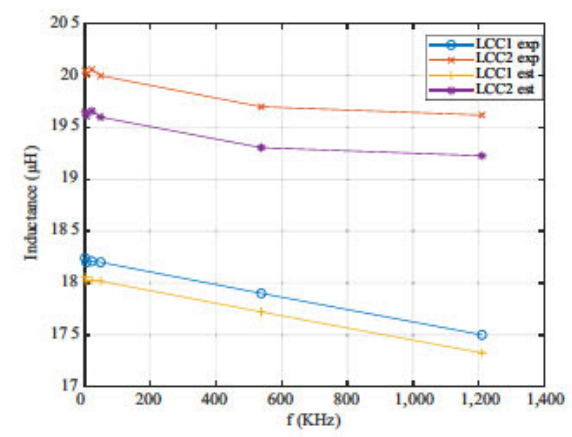

(f)
Figure 13.

FEMand experimental values for the polygonal model and the tested models
Notes: (a) FEA calculations (R1) of Component III; (b) FEA calculations (R1) of Component IV; (c) Test Results of Component III; (d) Test Results of Component IV; (e) FEA calculations (L) of Component I; and (f) FEA calculations (L) of Component II 
A parameter extraction procedure has been developed for $3 \mathrm{D}$ to obtain the resistance and inductance for an electrical equivalent circuit. The procedure contains two original contributions.

The first one is enclosed in the pre-modeling phase where the component simplification consists on the conductor cross-section modification (from circular to polygonal section) to reduce the $\mathrm{FE}$ and its correlative material conductivity adjustment to obtain the same values for the resistance and inductance at DC. It has been demonstrated that the hexagonal section for the conductor cross-section is the most effective simplification from the different polygonal options analyzed.

The second is allocated in the post-processing phase, after the parameter extraction based on energies, where a correction factor is needed for the extracted resistance from the Polygonal Model due to the different behavior of the high frequency effects in polygonal and circular cross-sections of the windings. The correction factor is the sum of two coefficients, one for the skin effect and the second for the proximity effect.

The coil inductance does not have to be necessarily recalculated using any correction factor at high frequencies due to fact, that the obtained value from the Polygonal Model is very close to value given by correction factor. The Polygonal Model permits the convergence in the simulation or a CPU time reduction about 95 per cent and by using the correction factor we reach very high accuracy (error $<5$ per cent) even for high frequencies. This new methodology brings great potential into the optimization process based on 3 D FEA.

Another of the main advantages of the proposed method is that enables the simulation of complex $3 \mathrm{D}$ models to study relevant effects in magnetic components as core saturation or thermal distribution.

\section{References}

Aljohani, O., Abu Siada, A. and Li, S. (2016), "High frequency power transformer modelling for frequency response analysis studies", 2016 International Conference on Condition Monitoring and Diagnosis (CMD). Xi' an. 2016, IEEE, Piscataway, NJ, pp. 291294.

Asensi, R., Prieto, R., Cobos, J.A. and Uceda, J. (2007), "Modeling high frequency multiwinding magnetic components using finite element analysis", IEEE Transactions on Magnetics, Vol. 43 No. 10, pp. 38403850.

Bednarz, C., Schreiber, H. and Leone, M.(2018), "Efficient multiport equivalent circuit and proximity effect in parallel conductors with arbitrary cross sections", IEEE Transactions on Electromagnetic Compatibility, Vol. 99.

Belevitch, V. (1971), "The lateral skin effect in a flat conductor", Philips tech. Rev 32.221 231. 1971. No.6/7.18.

Ben Messaoud, A., Talmoudi, S. and Ksouri Lahmar, M. (2016), "Multimodel approach for modeling of nonlinear systems: validities' optimal computation”, COMPEL The International Journal for Computation and Mathematics in Electrical and Electronic Engineering, Vol. 37 No. 1, pp. 153175.

Coulomb, J. L. (2014), "Framework for the optimization of online computable models", COMPEL The International Journal for Computation and Mathematics in Electrical and Electronic Engineering, Vol. 33 No. 3, pp. 745758.

Dias, R.A., Lira, G.R. S., Costa, E.G., Ferreira, R.S. and Andrade, A.F. (2018), "Skin effect comparative analysis in electric cables using computational simulations", 2018 Simposio Brasileiro de Sistemas Eletricos (SBSE), Niteroi, pp. 16. 
Dowell, P.L. (1966), "Effects of eddy currents in transformer windings", Proceedings of the Institution of Electrical Engineers, Vol. 113 No. 8, pp. 13871394.

Ferreira, J.A. (1994), "Improved analytical modeling of conductive losses in magnetic components", IEEE Transactions on Power Electronics, Vol. 9 No. 1, pp. 127131.

Gonzalez Teodoro, J.R., Asensi, R. and Prieto, R. (2015), "Simplifications in 3D high low frequency models of multiwinding magnetic components (EE and toroidal cores)", International Journal of Magnetics and Electromagnetism, Vol. 1 No. 1, p. 3.

Iatcheva, I. (2018), "Application of field modeling and determination of parameters in electrical engineering education”, COMPEL The International Journal for Computation and Mathematics in Electrical and Electronic Engineering, Vol. 37 No. 5, pp. 15851596.

Igarashi, H. (2017), "Semi analytical approach for finite element analysis of multi turn coil considering skin and proximity effects", IEEE Transactions on Magnetics, Vol. 53 No. 1, pp. 17.

Kiselev, D.S., Persova, M.G., Soloveichik, Y.G., Koshkina, Y.I., Vagin, D.V. and Simon, E.I. (2016), "Comparison of approaches and the software for 3D finite element modeling of harmonic electromagnetic fields", 2016 13th International Scientific Technical Conference on Actual Problems of Electronics Instrument Engineering (APEIE), 2016, Novosibirsk, pp. 255258.

Lu, M. and Ngo, K.D.T. (2017), "An analytical method to calculate winding resistance for planar coil with ferrite plate and litz wire in inductive power transfer", 2017 IEEE Energy Conversion Congress and Exposition (ECCE), Cincinnati, OH, pp. 111117.

Niyomsatian, K., Gyselinck, J.J.C. and Sabariego, R.V. (2018), "Experimental extraction of winding resistance in litz wire transformers influence of winding mutual resistancë", IEEE Transactions on Power Electronics, Vol. 34 No. 7, pp. 67366746.

Okamoto, Y., Masuda, H., Kanda, Y., Hoshino, R. and Wakao, S. (2016), "Improvement of topology optimization method based on level set function in magnetic field problem", COMPEL The International Journal for Computation and Mathematics in Electrical and Electronic Engineering, Vol. 37 No. 2, pp. 630644.

Phukan, R., Ravi, L., Tallam, R. and Shahirini, A. (2016), “A new high frequency simulation model for multi winding transformers used in switched mode power supplies", 2016 IEEE Energy Conversion Congress and Exposition (ECCE), 2016, Milwaukee, WI, pp. 16.

Zhang, H., Lee, J., Iyer, N.M. and Cao, L. (2017), "New analytical equations for skin and proximity effects in interconnects operated at high frequency", 2017 IEEE Electron Devices Technology and Manufacturing Conference (EDTM), Toyama, pp. 39 41, doi: 10.1109/EDTM.2017.7947499.

\section{Corresponding author}

Jorge Rafael González Teodoro can be contacted at: jordirgt@hotmail.com 